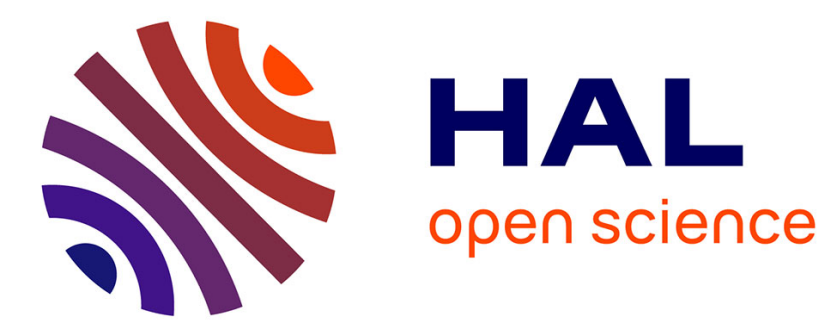

\title{
Une anomalie aortique
}

T Levasseur, Y N'guyen, C Aludaat, M Espinosa, D Metz

\section{To cite this version:}

T Levasseur, Y N'guyen, C Aludaat, M Espinosa, D Metz. Une anomalie aortique. La Revue de Médecine Interne, 2018, 39, pp.602 - 603. 10.1016/j.revmed.2016.10.382 . hal-03267280

\section{HAL Id: hal-03267280 \\ https://hal.science/hal-03267280}

Submitted on 29 Jun 2021

HAL is a multi-disciplinary open access archive for the deposit and dissemination of scientific research documents, whether they are published or not. The documents may come from teaching and research institutions in France or abroad, or from public or private research centers.
L'archive ouverte pluridisciplinaire HAL, est destinée au dépôt et à la diffusion de documents scientifiques de niveau recherche, publiés ou non, émanant des établissements d'enseignement et de recherche français ou étrangers, des laboratoires publics ou privés. 


\title{
Image
}

\section{La revue de médecine interne}

\section{Une anomalie aortique. An aortic abnormality. (1075 mots)}

\author{
T. Levasseur ; Y. N'Guyen ; C. Aludaat ; M. Espinosa ; D. Metz \\ ${ }^{\text {a }}$ Service de Cardiologie et maladies vasculaires, Hôpital Robert Debré, CHU Reims. \\ ${ }^{\text {b }}$ Service de Médecine interne, maladies infectieuses et Immunologie Clinique, Hôpital \\ Robert Debré, CHU Reims.
}

${ }^{\mathrm{c}}$ Service de Chirurgie Cardiothoracique, Hôpital Robert Debré, CHU Reims.

* auteur correspondant: Service de Cardiologie et maladies vasculaires, Hôpital Robert Debré, Avenue Général Koenig, 51100 Reims. Tel +33 3267863 15. Fax +33 326782887. Mail: tlevasseur@chu-reims.fr 
Résumé : aucun

Abstract: none

Mots clés : Clostridium, aortite infectieuse, aortite à anaérobies

Keywords: Clostridium, infectious aortitis, anaerobic aortitis 


\section{Histoire :}

Un homme de 71 ans ayant pour seul antécédent un infarctus antérieur avec angioplastie de l'artère inter-ventriculaire antérieure dix ans auparavant, toujours sous anti-agrégant, a été hospitalisé pour une douleur thoracique. Les douleurs évoluaient depuis la veille et semblaient positionnelles, majorées à l'inspiration profonde. Il y avait une diarrhée et des douleurs abdominales diffuses évoluant depuis 48 heures. L'électrocardiogramme retrouvait un susdécalage du segment ST dans le territoire inférieur isolé sans miroir. L'échocardiographie transthoracique retrouvait une fraction d'éjection du ventricule gauche estimée à $60 \%$ sans trouble de la cinétique notamment inférieur et surtout un épanchement péricardique centimétrique. On notait une dilatation modérée de l'aorte ascendante à $39 \mathrm{~mm}$ avec une insuffisance aortique de grade 1 sans argument en faveur d'une dissection aortique. La troponine ultrasensible plasmatique était à $29 \mathrm{ng} / \mathrm{l}(\mathrm{N}<14)$ tandis qu'il existait un syndrome inflammatoire très bruyant (CRP à $471 \mathrm{mg} / \mathrm{l}$ et de la PCT à $0.82 \mu \mathrm{g} / \mathrm{l})$ avec hyperleucocytose à polynucléaires neutrophiles $(18.7 \mathrm{G} / \mathrm{L})$. Un scanner thoracique initial retrouvait un aspect d'hématome intra-mural de l'aorte thoracique ascendante (figure 1A) inhabituel par son hétérogénéité. De ce fait, un scanner de contrôle a été réalisé 48 heures plus tard (figure 1) alors que des Bacilles Gram positifs étaient mis en évidence sur les hémocultures. 


\section{Diagnostic :}

Une aortite emphysémateuse hématogène secondaire à une bactériémie à Clostridium septicum.

\section{Commentaires:}

Dans cette observation, le diagnostic inhabituel d'aortite infectieuse a été confirmé au bout de 3 jours devant la présence de nombreuses bulles d'air au sein de d'une aorte thoracique ascendante dilatée [1]. Depuis le diagnostic précoce des endocardites avec l'échocardiographie et le recul de la syphilis, une très grande variété de micro-organismes a été impliquée: cocci Gram positifs (Staphylococcus aureus ou Streptococcus spp) comme Bacilles Gram négatifs (BGN) (e.g Salmonella spp et Campylobacter fetus), même si ces derniers sont plus souvent impliqués dans les aortites de l'aorte abdominale [1]. La présence de Bacilles Gram Positifs (BGP) au sein des hémocultures confirmait l'implication de Clostridium septicum dans l'aortite [1,2]. En effet, bien que Clostridium septicum soit un BGP anaérobie strict inconstant du tube digestif [2,3], son implication dans les aortites infectieuses a été clairement mise en évidence dans une quarantaine d'observations sur les 30 dernières années [2]. En l'absence d'inoculation directe ou de diffusion par contiguïté [1], le mécanisme physiopathologique de l'invasion de la paroi aortique reste débattu entre une greffe directe du germe sur lésion athéromateuse et une embolisation des vaso vasorum. Toutefois, une association forte entre les infections à Clostridium septicum et la découverte d'une pathologie néoplasique digestive déjà mise en évidence dans la littérature de longue date [2], limite les conclusions physiopathologiques que l'on pourrait tirer de la prédominance de cette infection chez les sujets âgés. De manière intéressante, l'association entre une aortite à Clostridium septicum et la présence d'une néoplasie colique dans 29 des 40 cas rapportés [2] incite à pratiquer une coloscopie au même titre que les endocardites infectieuses à Streptococcus bovis.

Toutefois, une telle enquête n'est pas systématiquement réalisée du fait de la mortalité spontanée importante de cette infection avec $25 \%$ de mortalité à 24 heures et $100 \%$ à 6 mois en l'absence de chirurgie [4]. Le plus souvent, le décès survient après rupture de l'aorte secondaire le plus souvent à une fragilisation anévrysmale rapide malgré antibiothérapie, le pronostic des aortites à BGN semblant plus sombre avec un risque de rupture de $72 \%$ [1]. Dans notre observation, l'antibiothérapie comprenait cefotaxime et métronidazole avec des séances d'oxygénothérapie hyperbare, par analogie avec les infections bactériennes à anaérobies des tissus mous, chez ce patient pour lequel toute possibilité d'intervention 
chirurgicale était inenvisageable en phase aiguë. En effet, la pose d'une prothèse aortique en urgence type Bentall et non d'une endoprothèse vasculaire du fait de la proximité de la valve aortique ici sur une paroi fragilisée par une aortite emphysémateuse semblait trop risquée. A notre connaissance, il n'y a qu'un cas rapporté dans la littérature d'intervention en urgence avec succès immédiat pour une aortite emphysémateuse à Clostridium septicum, compliquée d'un faux anévrysme anastomotique conduisant au décès du patient 3 mois plus tard [5]. Nous avons donc décidé d'attendre une amélioration de l'inflammation locale de la paroi artérielle sous antibiothérapie avant d'envisager une chirurgie mais le patient a présenté brutalement une asystolie probablement liée à une rupture de son aorte ascendante.

Conflit d'intérêts : aucun.

Sources de financement : aucun.

\section{Références :}

1. Revest M, Decaux O. Thoracic infectious aortitis : microbiology, pathophysiology and treatment. Rev Med Interne. 2007;28:108-15.

2. Shaha A, Yousuf T. Clostridium septicum aortitis of the infrarenal abdominal aorta. J clin Med Res. 2016;8:168-174

3. Moore WE, Holdeman LV. Human fecal flora : the normal flora of 20 Japanese-Hawaiians. Appl Microbiol. 1974 ;27:961-979.

4. Patel P.D, Arora RR. Pathophysiology, diagnosis and management of aortic dissection. Ther. Adv. Cardiovasc.Disc. 2008 ;2:39-468

5. Sailor DM, Eidt JF. Primary Clostridium septicum aortitis: a rare cause of necrotizing suprarenal aortic infection. A case report and review of the literature. J Vasc Surg. 1996;23:714-8. 


\section{Légende des figures :}

Figure 1 : Scanner thoracique en coupe frontale passant par l'aorte ascendante initial (A), après 3 jours : nombreuses bulles d'air au sein de l'aorte ascendante (B). 